\title{
Complementarity of energy resources for the electrical generation: a review
}

\author{
Angélica Vanessa Aldana Urrea - Diego Julián Rodríguez Patarroyob
}

\begin{abstract}
Resumen: ante las necesidades de aumentar los índices de cobertura eléctrica, generar medidas para la adaptación al cambio climático y diversificar la matriz energética de los países, se han desarrollado diversos estudios para establecer la complementariedad entre recursos energéticos. En este sentido, este documento presenta una revisión bibliográfica de algunas regiones a nivel mundial, para evidenciar las iniciativas investigativas alrededor del estudio de la complementariedad energética. Esta revisión incluye estudios que, además de establecer la complementariedad entre recursos solares, eólicos e hídricos, incluyen demanda, precios de electricidad, etcétera. De los resultados obtenidos se destaca que, si bien diversos estudios realizados en todo el mundo han demostrado que la mayor parte de la producción de sistemas de energía renovable presenta una naturaleza variable y, en parte, impredecible, hacer uso de más de un tipo de energía renovable mejora el rendimiento de estos sistemas, disminuye las fluctuaciones en la generación y aumenta la confiabilidad del sistema. Asimismo, se encuentra que el método más utilizado para la evaluación de complementariedad energética es el factor de correlación. Otros métodos incluyen simulaciones, aplicación de modelos y algoritmos de optimización. La información insumo para la realización de estos estudios proviene de los registros de los sistemas eléctricos de potencia de los países, de la aplicación de modelos de predicción meteorológica, de datos climatológicos históricos procedentes de satélites o de mediciones de estaciones meteorológicas, entre otros. Para Colombia se observan oportunidades de generar conocimiento alrededor de la complementariedad de los recursos energéticos.
\end{abstract}

Palabras clave: complementariedad energética; energía solar; energía eólica; energía hidráulica; energía renovable; seguridad energética.

Fecha recepción: 3 de septiembre de 2018 Fecha aprobación: 9 de marzo de 2019

Cómo citar: A. V. Aldana Urrea y D. J. Rodríguez Patarroyo, "Complementarity of energy resources for the electrical generation: a review," Ciencia e Ingenieria Neogranadina, vol. 29, no. 2, pp. 99-114, 2019. DOI: https://doi.org/10.18359/rcin.3625

a Universidad Distrital Francisco José de Caldas, Colombia.

Correo electrónico: angelica.aldanau@gmail.com. ORCID: https://orcid.org/0000-0003-4201-9319

b Universidad Distrital Francisco José de Caldas, Colombia.

Correo electrónico: djrodriguezp@gmail.com. ORCID: https://orcid.org/0000-0002-4907-5674 
Abstract: Given the needs to increase the rates of electricity coverage, to generate measures to adapt the Climate Change and to diversify the Matrix Energy in the countries, there have been developed a lot of studies to establish the complementarity of energy resources. In that sense, this document presents a literature review of some regions worldwide, to highlight the research initiatives around the study of energy complementarity. This review includes studies about establishing the complementarity of solar, wind and water resources, and includes demand, electricity prices, etc. Among the results found, it is noted that although several studies conducted around the world have shown that most of the renewable energy production systems has a variable and unpredictable nature, make use of more than one type of renewable energy improves the performance of those systems, it decreases the generation fluctuations and increases the system reliability. Also, it is found that the correlation factor is the most used method for the evaluation of energy complementarity. Other methods include simulations, model application, optimization algorithms, etc. In the same way, the input information for the realization of these studies comes from the registries of the electrical power systems of the countries, from the application of meteorological prediction models, from historical climatological data coming from satellites or from measurements of meteorological stations, among others. There are opportunities observed in Colombia to generate knowledge about complementarity between energy resources.

Keywords: Energy Complementarity; Solar Energy; Wind Energy; Hydraulic Energy; Renewable Energy; Energy Security. 


\section{Introducción}

Gracias a su posición geográfica, Colombia cuenta con una amplia oferta hídrica. El sector eléctrico colombiano tiene una capacidad efectiva de 16.420 MW, de los cuales el 69,8\% corresponde a generación hidroeléctrica, seguida por la generación a través de centrales térmicas con un $31 \%$, en promedio para 2015 [1].

Una característica del sistema eléctrico colombiano está relacionada con su alta variabilidad hidrológica, atribuida en buena medida al fenómeno de El Niño, que ocurre periódicamente de cada dos a siete años. Este fenómeno trae consigo prolongación de los periodos secos, lo que afecta directamente la disponibilidad de agua para la generación hidroeléctrica y, por consiguiente, el aumento en la generación térmica, las alzas en los precios de la energía y eventualmente los racionamientos de energía [2].

En este sentido, se ha identificado, además, que en épocas de El Niño, las regiones más afectadas con sequías son las mismas que están asociadas a las grandes plantas hidroeléctricas del país. En contraste, en las regiones donde se perciben aumentos de precipitaciones no existen proyectos de generación hidroeléctrica de gran envergadura.

Entre tanto, el sector eléctrico del país requiere minimizar su vulnerabilidad ante variaciones del clima y ante efectos del cambio climático, por medio de medidas de adaptación que permitan garantizar un ritmo positivo y sostenible de crecimiento a largo plazo [2]. Adicionalmente, y de acuerdo con la Ley 1715 de 2014 [3] y [4], es importante diversificar las fuentes de generación eléctrica en Colombia, con el fin de evitar un aumento significativo en emisiones de gases efecto invernadero (GEI) a 2025 (aumento del 100\%).

Por otra parte, y según la Unidad de Planeación Minero Energética (UPME) [5], para 2015 el Índice de Cobertura de Energía Eléctrica (ICEE) a nivel nacional era del 96,96\%, desagregada en urbano con un $99,72 \%$ y rural con un $87,83 \%$, respectivamente. Sin embargo, se identifican departamentos como Vichada con una cobertura inferior al $60 \%$, y Putumayo y Amazonas con coberturas inferiores al $65 \%$. Por su parte, la cobertura de electrificación en las zonas rurales del departamento de Cundinamarca está alrededor del $96 \%$.

Por consiguiente y con el objetivo de identificar la opción híbrida conformada por distintas fuentes energéticas como opción con alta probabilidad de cubrir los requerimientos de energía que un sistema pueda demandar a lo largo del año [6], resulta necesario caracterizar los recursos energéticos de las diferentes regiones del país y establecer la complementariedad entre ellos. Lo anterior se hace por medio del estudio del comportamiento estacional de los recursos energéticos para establecer en qué ubicaciones y en qué épocas del año se presentan, por ejemplo, los mayores índices de disponibilidad hídrica y los menores índices de disponibilidad solar o viceversa.

Así, el objetivo se centra en lograr la mayor distribución geográfica de las plantas renovables para generar respaldo de electricidad con otra fuente energética, al momento que falte o baje la intensidad de unos de los recursos con que se esté generando energía [7]. En este sentido, el objetivo de la presente revisión bibliográfica se centra en generar el estado del arte alrededor de la determinación de la complementariedad de recursos energéticos para la generación de electricidad en diferentes partes del mundo.

\section{Complementariedad energética para la generación eléctrica}

La mayor parte de la producción de sistemas de energía renovable presenta una naturaleza variable y un poco impredecible, debido a factores climáticos como radiación solar, temperatura del aire, velocidad del viento, precipitación, entre otros [8]. Por lo tanto, los sistemas de generación basados en fuentes renovables se enfrentan a dificultades de aceptación asociadas a costos iniciales y bajas eficiencias de funcionamiento. Una alternativa para mejorar el rendimiento es utilizar más de una fuente de energía renovable para proporcionar algún grado de complementariedad [9] [10]. La complementariedad entre estos recursos es un tema clave para el sistema, pues es posible que un sistema de generación conjunto basado en energía solar y eólica ahorre el costo de al menos un esquema de 
almacenamiento de energía; además, podría contribuir a la seguridad del sistema al disminuir las fluctuaciones en la generación [11], [12] y [13].

De acuerdo con [14], uno de los aspectos relevantes para alcanzar una mezcla de generación de electricidad menos contaminante está asociado a la intermitencia de las renovables. Al respecto, en [15] se menciona que una de las formas más prometedoras para reducir las fluctuaciones en la producción de energía con recursos renovables, específicamente de las energías eólica y solar, es tomar ventaja de su variabilidad espacial. En este sentido, el estudio de la complementariedad de recursos energéticos ha estado motivado principalmente por generar herramientas, métodos y estrategias que permitan planificar la expansión de la generación y promuevan la introducción de energías renovables no convencionales en los esquemas energéticos de los países. Ello en pro de diversificar las matrices energéticas y hacerlas más limpias, y así contribuir a un crecimiento económico sostenible y con los mínimos impactos al medio ambiente [16].

\section{Sus inicios}

Se han desarrollado diversas investigaciones en torno a la complementariedad de fuentes alternativas de energía; las primeras de estas se enfocaron en estudiar la confiabilidad de sistemas de generación basados en recurso eólico. Como muestra de ello, se menciona a [17] y [18], que indican que la confiabilidad de una fuente de generación basada en viento aumenta en la medida en que se sumen nuevas unidades de generación al sistema de potencia, lo cual hace que este sea más robusto y predecible. De igual manera, en [19] se demuestra que los requerimientos de contingencia pueden disminuir y la intermitencia propia del recurso eólico puede ser mitigada, hecho que aumenta el número de parques instalados.

A continuación, se muestra una revisión bibliográfica de algunas regiones a nivel mundial, para evidenciar las iniciativas investigativas alrededor del estudio de la complementariedad energética y el aprovechamiento de estas para contribuir a la diversificación de la matriz energética en diferentes países.

\section{Caso europeo}

Para el caso europeo se cuenta con estudios enfocados en estudiar la complementariedad energética entre los recursos eólico e hidráulico. Por ejemplo, para el sistema eléctrico nórdico se desarrolló una investigación basada en el modelo de programación de energía multiárea de EFI (EMPS -EFI’s Multiárea Power Scheduling Model, por sus siglas en inglés), para establecer los beneficios que puede percibir el sistema al incorporar energía eólica en la programación de la producción de energía hidroeléctrica. Al incorporar la producción de energía eólica en el modelo de programación de producción hidroeléctrica, se demostró que aumenta la cantidad de generación eólica, lo que se asocia con una mejor gestión de los depósitos de agua, lo que evita un derrame [20].

De igual manera y como ejemplo de un estudio en el que se evalúa la complementariedad entre recursos hídricos y recursos solares, se presenta el trabajo de [21], en el cual se emplea una metodología basada en un algoritmo de optimización para establecer el grado de complementariedad temporal entre pequeñas centrales hidroeléctricas y sistemas solares fotovoltaicos en Hungría. Para este estudio se emplearon registros de radiación solar de la instalación de aplicación de satélites para monitoreo del clima (CMSAF2) y datos hidrológicos procedentes de mediciones en la Estación de medición Sárvár gauging en el oeste de Hungría.

También se encuentran estudios que enfocan sus análisis en establecer la complementariedad energética entre recursos eólico y solar. Como muestra de ello se presenta el estudio de [11], que evalúa la complementariedad de los recursos solar y eólico para un año de prueba en Italia; la información espacial y temporal de los recursos solar y eólico proviene de los modelos PVGIs y MINNI. Asimismo, se desarrolla una simulación de Monte Carlo para estimar cómo las producciones de energía solar y eólica a gran escala podrían estar involucradas para complementarse en un escenario que incluye hasta 100 sitios de producción de energía en todo el país.

En Suecia, en [22] se muestra el coeficiente de correlación muestral entre la energía eólica y la energía solar por medio de un análisis basado en 
datos climáticos de una serie de tiempo de ocho años, con una resolución por hora. Como parte de los resultados de este estudio, se encuentra también el modelado de la energía solar y eólica a gran escala y la determinación de los efectos de la dispersión geográfica de los puntos de generación y de la combinación de estos dos recursos.

En ese orden de ideas, resulta interesante evaluar hasta qué punto una combinación de generación eólica y solar puede conducir a una carga estable. Como ejemplo de un estudio desarrollado desde esta óptica, se cita a [23], que evidencia el aprovechamiento del equilibrio espacio-temporal de estos recursos y las capacidades de almacenamiento de las plantas para la región de Andalucía en España. El análisis estadístico desarrollado incluye análisis de componentes principales (PCA) y análisis de correlación canónica (CCA) y los datos utilizados consisten en la velocidad del viento por hora y las estimaciones de radiación, obtenidos por medio del modelo numérico de predicción meteorológica (WRF).

Además, es posible mencionar otros de los estudios que involucran la demanda dentro de los análisis de complementariedad de recursos. Por ejemplo, en [24] se desarrolla un análisis por medio del coeficiente de correlación entre las generaciones de energía en parques solares fotovoltaicos y eólicos, y la carga en el sistema de energía Lituano, empleando el software Energy PRO. Otro ejemplo de esto se muestra en la investigación de [25], en la cual, por medio de modelos para estimar las series temporales de carga y producción hidroeléctrica y solar, se estiman los requerimientos de almacenamiento de este híbrido en una población que representa aproximadamente el $10 \%$ de la población italiana. El método utilizado incluye análisis de la desviación estándar del balance de carga y el coeficiente de correlación de Pearson. Los datos de consumo de electricidad en intervalos de una hora desde 2000 hasta 2010 fueron proporcionados por la red europea de operadores de sistemas de transmisión y la información de producción hidroeléctrica y solar se obtuvo a través de modelos.

También se encuentran enfoques más complejos. Casos como estos se observan por ejemplo en el análisis desarrollado por [26] para 27 países de Europa. Allí se evalúa la flexibilidad de un sistema de generación hidráulica y solar (capacidad para responder ante los cambios en la demanda y su oferta energética complementaria), basado en series temporales de producción de energía y datos de velocidad del viento e irradiancia solar de la NASA.

\section{Caso Asia y Oceanía}

En estas regiones del planeta también se encuentran estudios de complementariedad de recursos solares y eólicos; por ejemplo, la investigación de [27] evalúa la sinergia espacio temporal entre los recursos solares y eólicos en Australia, utilizando datos de viento y sol procedente del análisisretrospectivo de la era moderna para la investigación y aplicaciones (Modern Era Retrospective-analysis for Research and Applications [MERRA]). El análisis estadístico incluye el estudio de la variabilidad de los recursos, capturado en términos del coeficiente relativo de variación ( $\mathrm{RCoV}$ ) y del rango intercuartil (IQR). Además, se considera la cuantificación de la disponibilidad y persistencia del recurso, mediante el umbral de densidad de potencia. Por su parte, en Corea se desarrolló un estudio para determinar la correlación entre la energía eólica y solar en función del tiempo y la región (Isla de Jeju), con el objetivo de predecir con mayor exactitud la mezcla de generación que garantizara un suministro eléctrico estable [28].

Si bien el mayor porcentaje de países dependientes de los recursos hídricos para la generación eléctrica se concentran en América Latina, en países como la India o Nueva Zelanda se observa el mismo fenómeno. Incluso, se evidencian también dificultades sociales causadas por la construcción de grandes centrales hidroeléctricas y afectaciones en el suministro de energía en los periodos secos, debido a la escasez del recurso [29], y [30]. Tal situación motivó en India el desarrollo de una metodología capaz de encontrar la combinación óptima del híbrido fotovoltaico-eólico para cualquier región del mundo. El trabajo de [30] se concentra en un análisis detallado de los recursos solares y eólicos empleando diferentes fuentes de información: datos de la NASA, datos pronosticados de Artificial Neural Network (ANN), datos medidos y datos estimados. El modelo de optimización híbrida para energías renovables eléctricas (HOMER), 
desarrollado por el Laboratorio Nacional de Energía Renovable (NREL) de Estados Unidos, fue empleado para este análisis.

Asimismo, es posible mencionar estudios que involucran la demanda dentro de los análisis de complementariedad de recursos. Para Queensland, Australia del Sur y Tasmania se encontró una fuerte correlación entre la velocidad del viento y la demanda de electricidad, conclusión que se deriva de un estudio que empleó datos de velocidad del viento del Weather Research \& Forecasting Model y demanda de electricidad [31]. Los resultados son útiles además para determinar el tamaño de las fuentes de generación eólica que se requieren para satisfacer la demanda del Mercado Eléctrico Nacional de Australia (NEM, por sus siglas en inglés), obviando el almacenamiento de energía.

De igual manera, en Nueva Zelanda se elaboró un análisis de complementariedad energética entre los recursos eólicos e hídricos que incluye, además de la demanda, los precios de la electricidad [29]. Lo anterior, con el objetivo de comprender cuál sería el impacto en el sistema eléctrico nacional al aumentar la cantidad de generación eólica. El objetivo del estudio se centra en obtener información sobre las ventajas y desventajas que podría llevar consigo el desarrollo de la energía eólica en diferentes partes del país. Para este análisis se separan los patrones estacionales de las anomalías, y así se tuvo una visión más clara del comportamiento de la complementariedad energética considerando anomalías. Esto permite demostrar que en algunas ubicaciones son las anomalías las que tienen una correlación más significativa con los precios de electricidad, por encima de los patrones estacionales. Los autores sostienen que la metodología desarrollada es aplicable a cualquier sistema eléctrico que utilice recursos eólicos e hídricos, como Chile, Colombia y varias jurisdicciones de América del Norte y del Norte de Europa.

\section{Norteamérica}

Para el continente americano, específicamente Norte América, también se destacan investigaciones que tienen como objetivo central el estudio de la complementariedad de los recursos solares y eólicos. Por ejemplo, en Oklahoma se desarrolló un enfoque para calcular un índice de complementariedad entre radiación solar y viento sobre 127 sitios de Mesonet (red de estaciones meteorológicas automáticas). Se investigaron los impactos de diversos factores geográficos en la complementariedad de estos recursos; para ello se aplicaron análisis de componentes principales y modelos de regresión ponderados geográficamente [32].

En Ontario, Canadá, se evalúa el valor de aumentar el número de plantas de generación eólica y solar, tanto próxima como geográficamente dispersa [33], con el propósito de ver si es posible lograr una curva de producción de energía un poco más "suave". Es decir, disminuir los picos bajos y altos, en comparación con la curva de generación que produce una de estas tecnologías instaladas de forma individual. La información insumo para el desarrollo de esta investigación (mediciones síncronas y horarias de la radiación solar y las velocidades del viento) procede de los conjuntos de datos meteorológicos canadienses de energía e ingeniería de Canadá (cweeds). El análisis estadístico incluye: representación gráfica, un método de clasificación de percentiles y el desarrollo de un máximo teórico para una medición de capacidad.

Asimismo, se evidencian análisis que incluyen la demanda dentro de los estudios de complementariedad energética. En estos términos, se encuentra que en el estudio de [34] se cuantifica el grado de correlación entre la electricidad generada por plantas concentradoras de energía solar térmica (CSP) - la energía eólica y las CSP - y la demanda de electricidad. El trabajo fue desarrollado en 356 regiones de Estados Unidos, considerando información de insolación directa normal del satélite Pérez 2005, vientos por medio del modelo numérico de predicción meteorológica (NWP) de modelo de investigación y predicción del clima (WRF), y los datos de demanda por hora de 2005 a partir de datos de nivel de utilidad de Ventyx. Las correlaciones se calculan utilizando el coeficiente de correlación de Pearson de datos horarios sobre una base anual.

Además, la investigación [35] evalúa tres estrategias para mejorar la correlación entre la producción de energía fotovoltaica y la demanda. La primera de ellas consiste en optimizar la orientación de los módulos de generación, la segunda busca la 
combinación de matrices geográficamente dispersas y la tercera consiste en emplear un sistema de almacenamiento de energía. A partir de este trabajo fue posible establecer que las fuentes fotovoltaicas dispersas geográficamente y el almacenamiento de energía mejoran la correlación en estudio. Los datos empleados para esta investigación (irradiación horizontal global por hora [GHI], velocidad del viento y temperatura) provienen de MERRA.

\section{Latinoamérica}

Los sistemas energéticos que cuentan con una participación significativa de la generación hidroeléctrica usualmente cuentan también con una estacionalidad de precios que se asocia directamente al comportamiento cíclico de la hidrología, el cual tiene precios bajos en temporadas de lluvias y precios que por lo general ascienden en temporadas de baja hidrología. A menudo, el comportamiento estacional de algunas energías renovables no hidráulicas es complementario a las precipitaciones en muchos países de América Latina (es decir, a medida que uno de estos recursos disminuye, el otro tiende a aumentar) [36].

En la región, Brasil se muestra como pionero en el desarrollo de investigaciones relacionadas con la complementariedad de recursos. Dichos estudios se han motivado principalmente desde la necesidad de diversificar la matriz energética con fuentes complementarias a la energía hidráulica. Lo anterior, en razón a limitaciones físicas y ambientales que impiden la construcción de nuevas centrales hidroeléctricas con grandes embalses. De acuerdo con esto, el recurso eólico se presenta como el más susceptible de complementar la matriz energética debido a su complementariedad intrínseca (los vientos más intensos y regulares se presentan justamente en el periodo seco del año), en especial en la región nordeste, donde se localiza el mayor potencial eólico de Brasil [37].

En este sentido, también se menciona el trabajo de [38], en el cual se presenta el estudio de la complementariedad energética entre los recursos hídricos y eólicos por medio de modelos autorregresivos multivariados. Los datos de comportamiento de la velocidad, dirección y temperatura del viento procedentes de la Administración Nacional Oceánica y Atmosférica de los Estados Unidos (NOAA, por sus siglas en inglés) y los datos de flujos de entrada promedio mensual fueron provistos por el Operador Nacional del Sistema Eléctrico de Brasil (ons) y representan el flujo de entrada natural promedio del mes.

Asimismo, [39] evalúa el impacto de la integración de la energía eólica y las pequeñas centrales hidroeléctricas en la confiabilidad del sistema interconectado nacional de Brasil. Además de esto, por medio del coeficiente de correlación también evalúa la complementariedad entre la generación hidráulica, generación eólica y la carga. En dichos términos, se establece cómo esa correlación puede beneficiar la oferta para proyecciones de demanda futuras mediante el uso de la simulación secuencial de Monte Carlo. Además de las mejoras en la confiabilidad del sistema eléctrico derivadas de los esquemas híbridos de generación, en [40] se demuestra que es posible percibir beneficios por parte de algunos generadores eólicos al asociarse con productores hidroeléctricos, ya que se minimiza su exposición al riesgo en el mercado spot. Como parte de los resultados de este análisis, se encuentra también la obtención de la mezcla de generación óptima y la cantidad de asignación del volumen de energía en contratos por medio de un algoritmo genético, modelando el valor del riesgo condicional (CVaR) como una medida de riesgo. Los datos de viento utilizados para desarrollar este estudio se consiguieron con el modelo vestas meso-escala y la NOAA. Además, utilizando series históricas de condiciones hidrológicas y ejecutando el modelo de Newave, se obtuvieron 61 series de precios de electricidad desde enero de 2013 hasta diciembre de 2020, mensualmente.

En otros países como Perú y México también se han desarrollado análisis similares. La investigación [16] desarrolla una metodología que permite localizar en orden jerárquico las mejores áreas para la instalación de parques de generación eólicos, basado en un análisis de complementariedad con los recursos hídricos, empleando un análisis secuencial de correlación combinatoria hídrica-eólica. Entre tanto, el trabajo [41] muestra cómo la energía eólica puede ser complementada con energía hidráulica. Para ello se emplean dos 
instalaciones hipotéticas como caso de estudio: una hidroeléctrica y otra eólica, las dos ubicadas en una región de México rica en recurso hídrico y eólico. Por medio del análisis del rendimiento del sistema híbrido, teniendo en cuenta los factores de capacidad, se muestra que es posible garantizar continuidad del servicio eléctrico empleando la energía hidráulica para compensar las fluctuaciones del viento. Se evidenció que el sistema híbrido es capaz de entregar cerca de 20 MW de energía firme al sistema de distribución eléctrica.

También se han identificado análisis que involucran en estudio de la complementariedad de recursos hídricos, frente a los recursos solares. Es el caso, por ejemplo, de una investigación desarrollada en Brasil [42] que propone el concepto de límite de rendimiento teórico para plantas de generación hidroeléctrica y solar, que está basado en simulaciones por computadora que utilizan funciones de disponibilidad de energía idealizadas. A partir de allí se estudian los efectos de los diferentes grados de complementariedad en el tiempo de los recursos hídrico y solar, en el rendimiento de un esquema híbrido de generación eléctrica. De esta manera, se encontró que los menores índices de falla (lo cuales miden el suministro de energía) están asociados a los mejores grados de complementariedad.

Iniciativas que buscan estudiar la complementariedad de recursos eólicos y solares para la región latinoamericana se han encontrado en países como Brasil y Uruguay. En el estudio [43], se analizan las correlaciones y correlaciones cruzadas en series temporales de radiación solar y velocidad del viento en la Isla Fernando de Noronha, ubicada al noreste de Brasil (datos proporcionados por el Centro Nacional de Previsiones de Tiempo y Estudios Climáticos (СРтEC) del Instituto Nacional de Investigaciones Espaciales de Brasil. Para ello se emplean métodos de análisis de fluctuación y correlación cruzada. Adicionalmente, en el trabajo [9] se evalúa la complementariedad entre la energía hídrica y la energía solar en el sur de Brasil. Para ello se empleó un índice adimensional en la misma o en ubicaciones distintas, y se consideraron datos sobre la disponibilidad de agua y de energía solar, publicados por la Fundación Estadual de Investigaciones Agropecuarias.
En Uruguay se cuenta con un informe resultado de un proyecto de cooperación entre la Agencia Española de Cooperación Internacional para el Desarrollo (AECID) y el Ministerio de Industria, Energía y Minería (MIEM), enmarcado en el "Programa de cambio climático - Promoción de fuentes renovables y uso eficiente de la energía" [44]. Con ayuda de información procedente de estaciones meteorológicas, este estudio presenta modelos estocásticos del recurso solar y eólico, que son aptos para la planificación de la generación eléctrica con la herramienta SimSEE. Estos modelos captan la dependencia estadística de los recursos en estudio con el ciclo anual y diario. Como continuación del trabajo anterior, se desarrolló un análisis de complementariedad de los recursos eólico y solar para su utilización en la generación eléctrica en gran escala. Adicionalmente, la investigación se enfoca aún más en el modelado, y con esto se logra el modelo estocástico CEGH eólico, solar y temperatura conjunto y además; un estudio de impacto sobre el uso de la conexión a la red de plantas mixtas solar y eólica [45].

Como muestra de una investigación que incluye dentro de su análisis de complementariedad recursos hídricos, solares y eólicos, se tiene en cuenta el trabajo [10], que evalúa la complementariedad de las pequeñas centrales hidroeléctricas, de los paneles fotovoltaicos y los parques eólicos en Río de Janeiro. Allí se emplea el coeficiente de correlación de Pearson basado en la radiación solar y velocidades del viento procedentes del Instituto Nacional de Investigaciones Espaciales (INPE, por sus siglas en inglés), y los datos de flujo de los ríos de la Agência Nacional de Águas (ANA, por sus siglas en inglés). Además, hace uso de la programación lineal para obtener la mezcla renovable óptima.

\section{Caso colombiano}

Para el caso colombiano se han encontrado estudios que desarrollan análisis de complementariedad entre recursos hídricos y eólicos, y que parten principalmente de la necesidad de diversificar la matriz energética del país. Uno de ellos corresponde al trabajo llevado a cabo por [46], quien desarrolla un análisis de complementariedad de estos recursos a partir de información diaria y mensual de series de caudales y velocidades de vientos a diferentes 
alturas de medición. Los datos proceden de estaciones del Instituto de Hidrología, Meteorología y Estudios Ambientales (Ideam) y de Isagen, ubicadas en la costa Caribe, y el análisis de correlación se desarrolló con el coeficiente de correlación de Pearson. El estudio determinó que el ciclo anual de la velocidad del viento está relacionado con los periodos de precipitación y sequía que se dan en la región, influenciados, entre otras cosas, por la zona de convergencia intertropical, que actúa sobre todo el territorio nacional.

Asimismo, se menciona el trabajo de [47], que emplea la teoría de portafolios (costos) para desarrollar un análisis de complementariedad en fuentes hidráulica y eólica para la generación eléctrica. En dicha investigación, por medio de un modelo de optimización, se calculan las fronteras eficientes de los recursos predominantes de generación de energía en combinación con la energía eólica, aprovechando las potencialidades de viento en el departamento de La Guajira. El análisis estuvo apoyado en mediciones horarias del viento por periodos diarios, mensuales y anuales procedentes de la estación Puerto Bolívar, y en el uso de los modelos de costos planteados por la UPME y el Energy Sector Management Assitance Program (ESMAP). Este documento deja ver que la energía eólica puede formar parte del conjunto de alternativas de generación de electricidad en el país, ya que participó en las fronteras eficientes de las diferentes combinaciones de portafolios propuestos.

Otras investigaciones también se han enfocado en el estudio de la complementariedad energética entre el recurso eólico y el hidráulico de la región costera del norte del país, específicamente en La Guajira [48] y [49]. En [48], por ejemplo, se demuestra la existencia de complementariedad energética entre recursos hídricos y eólicos, más que nada hablando de la producción de energía eólica durante los meses cuando se presenta el fenómeno de El Niño. Los autores aseguran que este análisis podría consolidarse como una justificación para el desarrollo de una nueva metodología de energía firme para el cargo por confiabilidad (ENFICC). Los análisis desarrollados incluyen factores de correlación entre series de viento y datos de producción hidroeléctrica procedentes de la UPME.
Finalmente, vale la pena mencionar los trabajos de [50] y [7], en los cuales se han generado estudios de complementariedad entre los recursos hídricos, eólicos y solares a nivel nacional. En [7], por ejemplo, se muestran oportunidades de instalar parques eólicos y plantas solares en sitios donde se presenta correlación de recursos, a fin de servir de respaldo para las centrales hidroeléctricas durante periodos de baja hidrología. El método empleado corresponde al factor de correlación de Pearson, y los datos que se usaron fueron procedentes del MERra, de la Oficina Global de Modelado y Asimilación (Global Modeling and Assimilation Office [GMAO]), de la NASA, XM y UPME.

\section{Visión general}

Con el fin de hacer una comparación entre los trabajos realizados en tópicos sobre complementariedad energética discriminado por regiones, la procedencia de los datos y los métodos utilizados para analizar la correlación entre los energéticos, se elabora la Tabla 1, que facilita este objetivo.

\section{Oportunidades de investigación}

Según la upMe [5], para 2015 el ICEE a nivel nacional es de 96,96\%, desagregado en: urbano con un $99,72 \%$ y rural con un $87,83 \%$; sin embargo, existen departamentos como Vichada que tienen una cobertura inferior al $60 \%$. Por su parte, la cobertura de electrificación en las zonas rurales del departamento de Cundinamarca está alrededor del $96 \%$.

En particular, para las zonas rurales de Cundinamarca aún se encuentran áreas donde se requiere suministrar el servicio de energía eléctrica. Además, zonas donde, si bien existe cobertura del servicio, se encontraron dificultades de calidad de la energía más acentuadas, sobre todo en las colas de distribución eléctrica [51]. Asimismo, se identifica la necesidad de establecer cómo se correlacionan los recursos energéticos disponibles en las zonas rurales de este departamento $y$, en función de esta información, establecer cómo se puede hacer uso de estos recursos para dar solución a las diferentes necesidades energéticas de la población rural. 
Tabla 1. Visión general referencias bibliográficas

\begin{tabular}{|c|c|c|}
\hline País & Datos y su procedencia & Método empleado \\
\hline Noruega & $\begin{array}{l}\text { Información de producción de energía eólica y programación de la } \\
\text { producción de energía hidroeléctrica del sistema eléctrico nórdico. }\end{array}$ & $\begin{array}{l}\text { Modelo de programación de energía } \\
\text { multiárea de EFI, (EMPS -EFI's Multiarea Power } \\
\text { Scheduling Model. }\end{array}$ \\
\hline Hungría & $\begin{array}{l}\text { Registros de radiación solar de la instalación de aplicación de } \\
\text { satélites para monitoreo del clima (CMSAF) y datos hidrológicos } \\
\text { procedentes de mediciones en la estación de medición Sárvár } \\
\text { gauging en el oeste de Hungría. }\end{array}$ & Algoritmo de optimización. \\
\hline Italia & $\begin{array}{l}\text { La información espacial y temporal de los recursos solar y eólico } \\
\text { proviene de los modelos (PVGIS y MINNI). }\end{array}$ & Simulación de Monte Carlo. \\
\hline Suecia & $\begin{array}{l}\text { Se utilizan series de datos de potencia de salida para unidades de } \\
\text { generación eólica y solar que cubren ocho años consecutivos con } \\
\text { una resolución por hora, modelada a partir de datos climáticos } \\
\text { históricos. }\end{array}$ & Coeficiente de correlación muestral. \\
\hline España & $\begin{array}{l}\text { Los datos utilizados en este trabajo consisten en la velocidad del } \\
\text { viento por hora y las estimaciones de radiación, obtenidos por } \\
\text { medio del WRF. }\end{array}$ & $\begin{array}{l}\text { Análisis de Componentes Principales (PCA) y } \\
\text { Análisis de Correlación Canónica (CCA). }\end{array}$ \\
\hline $\begin{array}{l}\text { República de } \\
\text { Lituania }\end{array}$ & $\begin{array}{l}\text { La información de generación de energía en parques solares } \\
\text { fotovoltaicos y eólicos, y la carga se obtuvieron del sistema de } \\
\text { energía Lituano. }\end{array}$ & Coeficiente de correlación muestral. \\
\hline Italia & $\begin{array}{l}\text { Los datos de consumo de electricidad en intervalos de una hora } \\
\text { desde } 2000 \text { hasta 2010, fueron proporcionados por la red europea } \\
\text { de operadores de sistemas de transmisión. La información de } \\
\text { producción hidroeléctrica y solar se obtiene por medio de modelos } \\
\text { que permiten estimar las series de tiempo de carga de energía y la } \\
\text { producción de energía hidroeléctrica y solar. }\end{array}$ & $\begin{array}{l}\text { Análisis de la desviación estándar del balance } \\
\text { de carga y el coeficiente de correlación de } \\
\text { Pearson. }\end{array}$ \\
\hline $\begin{array}{l}27 \text { países de } \\
\text { Europa }\end{array}$ & $\begin{array}{l}\text { Los datos de velocidad del viento e irradiancia solar provienen de } \\
\text { la NASA. }\end{array}$ & $\begin{array}{l}\text { Evaluación de los requisitos de flexibilidad } \\
\text { en los sistemas de energía futuros con altas } \\
\text { participaciones de generación variable. }\end{array}$ \\
\hline Australia & $\begin{array}{l}\text { Los datos de viento y sol proceden del análisis-retrospectivo del } \\
\text { MERRA. }\end{array}$ & $\begin{array}{l}\text { Coeficiente relativo de variación (RCOV) y del } \\
\text { rango intercuartil (IQR). Además, se considera } \\
\text { la cuantificación de la disponibilidad y } \\
\text { persistencia del recurso, mediante el umbral } \\
\text { de densidad de potencia. }\end{array}$ \\
\hline Corea & $\begin{array}{l}\text { Se empleó información de velocidades del viento y la cantidad de } \\
\text { luz solar de las regiones de la Isla de Jeju. }\end{array}$ & Coeficiente de correlación. \\
\hline India & $\begin{array}{l}\text { Para el sistema de generación híbrido fotovoltaico- eólico, se } \\
\text { emplearon datos de la NASA, datos pronosticados de ANN, datos } \\
\text { medidos y datos estimados. }\end{array}$ & El HOMER. \\
\hline $\begin{array}{l}\text { Queenslan, } \\
\text { Australia del } \\
\text { Sur y Tasmania }\end{array}$ & $\begin{array}{l}\text { Se emplean datos de demanda de electricidad de media hora y los } \\
\text { perfiles de velocidad del viento de tres años calendario 2010, } 2011 \\
\text { y 2012, los cuales proceden del Weather Research \& Forecasting } \\
\text { Model. }\end{array}$ & Coeficiente de correlación. \\
\hline Nueva Zelanda & $\begin{array}{l}\text { Los datos utilizados provienen de dos fuentes diferentes: por un } \\
\text { lado, el Conjunto de Datos Central de la Autoridad de Electricidad } \\
\text { de Nueva Zelanda, la cual proporcionó datos reales de producción } \\
\text { de energía eólica, datos de demanda, precios de electricidad y } \\
\text { registros sobre los niveles de almacenamiento hidráulico; por otro } \\
\text { lado, el Instituto Nacional de Agua y Atmósfera Research (NIWA), } \\
\text { que proporcionó datos sintéticos de velocidad del viento. }\end{array}$ & Coeficiente de correlación. \\
\hline
\end{tabular}


País

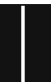

Oklahoma

La información asociada a radiación solar y viento, se obtuvo sobre 127 sitios de Mesonet.

La información de mediciones síncronas y horarias de la radiación

Canadá solar y las velocidades del viento, procede de los Conjuntos de Datos Meteorológicos Canadienses de Energía e Ingeniería de Canadá (cweeds).
Método empleado

Análisis de componentes principales y modelos de regresión ponderados geográficamente.

Análisis estadístico que incluye: representación gráfica, un método de clasificación de percentiles y el desarrollo de un máximo teórico para una medición de capacidad.

\begin{tabular}{|c|c|c|}
\hline $\begin{array}{l}356 \text { regiones } \\
\text { de los Estados } \\
\text { Unidos }\end{array}$ & $\begin{array}{l}\text { Los datos de las plantas concentradoras de energía solar térmica, } \\
\text { se originaron a partir de los datos de insolación directa normal del } \\
\text { satélite Pérez } 2005 \text { por hora en una escala de } 10 \mathrm{~km} \text {. Por su parte, la } \\
\text { información de vientos se obtuvo por medio del NWP de modelo de } \\
\text { investigación y predicción del clima (WRF). Finalmente, los datos de } \\
\text { demanda por hora de } 2005 \text { se obtuvieron a partir de datos de nivel } \\
\text { de utilidad de Ventyx. }\end{array}$ & Coeficiente de correlación. \\
\hline Canadá & $\begin{array}{l}\text { Los datos de irradiación horizontal global por hora (GHI), velocidad } \\
\text { del viento y temperatura, proceden del análisis retrospectivo del } \\
\text { MERRA. }\end{array}$ & Coeficiente de correlación. \\
\hline Brasil & $\begin{array}{l}\text { Los registros hídricos y eólicos, proceden de la NOAA, y los datos de } \\
\text { flujos de entrada promedio mensual, del ONS. }\end{array}$ & Modelos autorregresivos multivariados. \\
\hline Brasil & $\begin{array}{l}\text { Información de la generación hidráulica, generación eólica y la } \\
\text { carga, es obtenida sistema eléctrico de potencia de Brasil. }\end{array}$ & Simulación secuencial de Monte Carlo. \\
\hline Brasil & $\begin{array}{l}\text { Los datos del viento fueron obtenidos por el modelo cestas } \\
\text { meso-escala y NOAA). Utilizando series históricas de condiciones } \\
\text { hidrológicas y ejecutando el Modelo de Newave, se obtuvieron } \\
61 \text { series de precios de electricidad desde enero de } 2013 \text { hasta } \\
\text { diciembre de 2020, mensualmente. }\end{array}$ & Algoritmo genético. \\
\hline Perú & $\begin{array}{l}\text { La información utilizada incluye: } \\
\text { - Base de datos de viento. } \\
\text { - Modelos de previsión de viento. } \\
\text { - Base de datos de caudales hídricos de hidroeléctricas existentes. } \\
\text { - Modelos de previsión de recursos hídricos. } \\
\text { - Base de datos de tecnologías eólicas. } \\
\text { - Base de datos de tecnologías hídricas (para lugares de instalación } \\
\text { de centrales hidroeléctricas a nivel de agua). }\end{array}$ & $\begin{array}{l}\text { Análisis secuencial de correlación } \\
\text { combinatoria hídrica-eólica. }\end{array}$ \\
\hline México & $\begin{array}{l}\text { Se emplean instalaciones hipotéticas de energía eólica y energía } \\
\text { hidráulica como caso de estudio. }\end{array}$ & $\begin{array}{l}\text { Análisis del rendimiento del sistema híbrido, } \\
\text { teniendo en cuenta los factores de capacidad. }\end{array}$ \\
\hline Brasil & $\begin{array}{l}\text { De un sistema de generación híbrido se obtiene la información de } \\
\text { recursos hídricos y recursos solares. }\end{array}$ & $\begin{array}{l}\text { Simulaciones por computadora que utilizan } \\
\text { funciones de disponibilidad de energía } \\
\text { idealizadas. }\end{array}$ \\
\hline Brasil & $\begin{array}{l}\text { Las series temporales de radiación solar y velocidad del viento } \\
\text { forman parte de una base de datos climáticos proporcionada por el } \\
\text { CPTEC del Instituto Nacional de Investigaciones Espaciales de Brasil } \\
\text { (Instituto Nacional de Investigación Espacial). }\end{array}$ & $\begin{array}{l}\text { Métodos de análisis de fluctuación y } \\
\text { correlación cruzada. }\end{array}$ \\
\hline Brasil & $\begin{array}{l}\text { Se consideraron datos sobre la disponibilidad de agua y la } \\
\text { disponibilidad de energía solar, publicados por la Fundación } \\
\text { Estadual de Investigaciones Agropecuarias. }\end{array}$ & $\begin{array}{l}\text { Índice de complementariedad adimensional } \\
\text { en la misma o ubicaciones distintas. Este } \\
\text { índice considera } \\
\text { la diferencia de fase entre los valores } \\
\text { de disponibilidad de energía de las dos } \\
\text { fuentes, la relación entre las amplitudes de } \\
\text { variación de las funciones de disponibilidad } \\
\text { y la relación entre los dos valores de } \\
\text { disponibilidad promedio. }\end{array}$ \\
\hline
\end{tabular}


País

\begin{tabular}{|c|c|}
\hline Pais & Datos y su procedencia \\
\hline Uruguay & $\begin{array}{l}\text { La información histórica de datos de viento cons } \\
\text { medición, información obtenida de siete estacio } \\
\text { el territorio. }\end{array}$ \\
\hline Uruguay & Se emplea la misma información del trabajo ant \\
\hline Brasil & $\begin{array}{l}\text { Los registros de radiación solar y velocidades de } \\
\text { del INPE, y los datos de flujo de los ríos de la ANA. }\end{array}$ \\
\hline Colombia & $\begin{array}{l}\text { La información diaria y mensual de series de cau } \\
\text { de vientos a diferentes alturas de medición, proc } \\
\text { del Ideam e Isagen, ubicadas en la costa Caribe. }\end{array}$ \\
\hline Colombia & $\begin{array}{l}\text { El análisis estuvo apoyado en mediciones horari } \\
\text { periodos diarios, mensuales y anuales proceden } \\
\text { Puerto Bolívar, y en el uso de los modelos de cos } \\
\text { la UPME y el ESMAP. }\end{array}$ \\
\hline Colombia & $\begin{array}{l}\text { Las series de viento y los datos de producción hi } \\
\text { proceden de la UPME. }\end{array}$ \\
\hline Colombia & $\begin{array}{l}\text { En este estudio se utilizaron datos mensuales de } \\
\text { asociados con centrales hidroeléctricas, y datos } \\
\text { estaciones en el Caribe colombiano del Ideam. }\end{array}$ \\
\hline Colombia & $\begin{array}{l}\text { Los registros empleados son procedentes del ME } \\
\text { la NASA, además de XM y UPME. }\end{array}$ \\
\hline Colombia & $\begin{array}{l}\text { Los registros de recursos hídricos, eólicos y solare } \\
\text { proceden de XM, UPME, Ideam y Empresas Pública }\end{array}$ \\
\hline $\begin{array}{l}\text { De es } \\
\text { lidad, el } \\
\text { porales y } \\
\text { recursos } \\
\text { zonas rur } \\
\text { podrán b } \\
\text { rural y es } \\
\text { de baja d } \\
\text { tribuir co } \\
\text { política y } \\
\text { generació } \\
\text { se adapte } \\
\text { y tempor }\end{array}$ & $\begin{array}{l}\text { lanera, el conocimiento de la variabi- } \\
\text { uportamiento, las características tem- } \\
\text { complementariedad de los diferentes } \\
\text { rgéticos con los que se cuenta en las } \\
\text { del departamento de Cundinamarca } \\
\text { dar oportunidades de electrificación } \\
\text { mas de respaldo durante temporadas } \\
\text { nibilidad de recursos. Además, con- } \\
\text { ementos de juicio para dar señales de } \\
\text { léctrica no convencionales que mejor } \\
\text { las condiciones climáticas, geográficas } \\
\text { de la zona [7]. }\end{array}$ \\
\hline
\end{tabular}

\section{Conclusiones}

De acuerdo con la revisión bibliográfica realizada, se observó que, si bien diversos estudios realizados en todo el mundo han demostrado que la mayor parte de la producción de sistemas de energía renovable

\section{Método empleado}

Modelos estocásticos del recurso solar y eólico, los cuales son aptos para la planificación de la generación eléctrica con la herramienta SimSEE

Modelo estocástico CEGH eólico, solar y temperatura conjunto.

Coeficiente de correlació.n

Coeficiente de correlación.

Teoría de portafolios (costos), con un modelo de optimización.

Coeficiente de correlación.

Coeficiente de correlación.

Coeficiente de correlación.

Análisis cualitativo. presenta una naturaleza variable y en parte impredecible, hacer uso de más de un tipo de energía renovable mejora el rendimiento de estos sistemas, disminuye las fluctuaciones en la generación y aumenta la confiabilidad del sistema al adquirir algún grado de complementariedad.

En este sentido, para diferentes partes del mundo se encontraron estudios que, además de establecer la complementariedad entre recursos, incluyen demanda, precios de electricidad y análisis de los efectos de la dispersión geográfica de los puntos de generación. De igual forma, combinación de los recursos para la generación de electricidad, capacidad para responder ante cambios en la demanda y esquemas que permiten establecer el tamaño de las fuentes de generación o de sistemas de almacenamiento de energía que se requieren para satisfacer la demanda.

De las investigaciones encontradas para Europa, Asia, Oceanía y Norteamérica, se observa que el 
$47 \%$ de ellas emplean dentro de sus métodos el análisis por coeficiente de correlación. Mientras que dentro de las técnicas restantes se encuentra la utilización de otros métodos estadísticos como análisis de componentes principales, análisis del coeficiente relativo de desviación y del rango intercuartil, clasificación de percentiles, entre otros. Asimismo, se evidencia el uso de simulaciones, aplicación de modelos y algoritmos de optimización para los análisis de complementariedad energética.

La información insumo para la realización de estos estudios proviene de los registros de los sistemas eléctricos de potencia de los países, de la aplicación de modelos de predicción meteorológica, de datos climatológicos históricos procedentes de información satelital o de mediciones de estaciones meteorológicas. Además, en algunos casos se hace uso de modelos que permiten estimar las series de tiempo de carga de energía y la producción de energía hidroeléctrica y solar.

En América Latina se encuentra el mayor porcentaje de países que poseen matrices de generación eléctrica basadas en recursos hídricos. Se ha evidenciado que los estudios de complementariedad energética que se han desarrollado en la región han sido motivados por la necesidad de diversificar las matrices de generación. En este sentido, en la mayor parte de la literatura consultada se encontró que predominan los estudios de complementariedad del recurso hídrico con el recurso eólico.

Así, para esta región del mundo se encuentra que en el 35\% de los estudios se realiza evaluación por coeficiente de correlación. Otros métodos empleados incluyen simulaciones, algoritmos genéticos, análisis de rendimiento y requerimientos de flexibilidad, aplicación de modelos estocásticos, entre otros.

Se encontró además que una buena parte de los estudios basan sus análisis en los datos recolectados de estaciones meteorológicas terrestres, de la NASA o de entidades que se encargan del monitoreo de variables climatológicas de los países. Otro grupo de estudios incluye información de los sistemas eléctricos de cada país, fuentes de generación ficticias o modelamiento matemático para describir el comportamiento de los recursos.

Para Colombia se advierten oportunidades de generar conocimiento alrededor de la complemen- tariedad de los recursos energéticos presentes en las zonas rurales del departamento de Cundinamarca, incluso considerando la demanda. Esto como una herramienta con potencial para contribuir a la solución a las diferentes necesidades energéticas de la población rural e incluir fuentes renovables de energía en su esquema de generación eléctrica.

\section{Referencias}

[1] XM Expertos en Mercados, "Informe de Operación del SIN y Administración del Mercado - Generación del SIN," 2017. [En line]. Disponible en: http://informesanuales.xm.com.co/2015/SitePages/operacion/2-4-Generación-del-SIN.aspx

[2] Unidad de Planeación Minero Energética (UPME), "Estudio para determinar la vulnerabilidad y las opciones de adaptación del sector energético colombiano frente al cambio climático,” 2013. [En línea]. Disponible en: http://wwwl.upme.gov.co/InformacionCifras/ Documents/vulnerabilidad_opciones_adaptacion_ sector_energetico_colombiano_frente_cambio_climatico.pdf

[3] Congreso de Colombia, "Ley N ${ }^{\circ} 1715$ del 13 de mayo de 2014," Upme, no. May, p. 26, 2014.

[4] Fedesarrollo y Centro de Investigación Económica y Social, "Análisis costo beneficio de energías renovables no convencionales en Colombia," 2013. [En línea]. Disponible en: https://www.repository.fedesarrollo.org.co/ bitstream/handle/11445/331/Repor_Octubre_2013_ Garcia_et_al.pdf?sequence $=3 \&$ isAllowed $=y$

[5] Unidad de Planeación Minero Energética (UPME), "Plan Indicativo de Expansión de Cobertura de Energía Eléctrica (PIEC) 2016-2020,” pp. 0-48, 2016. [En línea]. Disponible en: http://www.upme.gov.co/ Siel/Siel/Portals/0/Piec/PIEC_2016-2020_PublicarDic202016.pdf

[6] J. Serván, "Análisis técnico-económico de un sistema híbrido de baja potencia eólico solar conectado a la red," Tesis de pregrado, Facultad de Ingeniería universidad de Piura, Perú, 2014.

[7]J. R. Paredes y J. J. Ramírez C. Energías renovables variables y su contribución a la seguridad energética: complementariedad en Colombia, Banco Interamericano de Desarrollo, 2017.

[8] K. Engeland, M. Borga, J.-D. Creutin, B. François, M.H. Ramos y J.-P. Vidal, "Space-time variability of climate variables and intermittent renewable electricity production - A review," Renewable and Sustainable Energy Reviews, vol. 79, pp. 600-617, 2017. DOI: https:// doi.org/10.1016/j.rser.2017.05.046 
[9] A. Beluco, P. K. de Souza y A. Krenzinger, "A dimensionless index evaluating the time complementarity between solar and hydraulic energies," Renew. Energy, vol. 33, no. 10, pp. 2157-2165, 2008. DOI: https://doi. org/10.1016/j.renene.2008.01.019

[10] C. de Oliveira Costa Souza Rosa, K. A. Costa, E. da Silva Christo y P. B. Bertahone, "Complementarity of hydro, photovoltaic, and wind power in Rio de Janeiro State," Sustain, vol. 9, no. 7, pp. 1-12, 2017. DOI: https:// doi.org/10.3390/su9071130

[11] F. Monforti, T. Huld, K. Bódis, L. Vitali, M. D’Isidoro y R. Lacal-Arántegui, “Assessing complementarity of wind and solar resources for energy production in Italy. A Monte Carlo approach," Renewable Energy, vol. 63, pp. 576-586, 2014. DOI: https://doi.org/10.1016/j. renene.2013.10.028

[12] L. Xu, X. Ruan, C. Mao, B. Zhang y Y. Luo, “An Improved Optimal Sizing Method for Wind-Solar-Battery Hybrid Power System," IEEE Transactions on Sustainable Energy, vol. 4, no. 3, pp. 774-785, 2013. DOI: https:// doi.org/10.1109/TSTE.2012.2228509

[13] A. Ademovic y M. Music, "Compatibility of Wind and Solar Power Generation in Reducing Effects of Power Output Intermittency - Case Study," en 2014 IEEE International Energy Conference (ENERGYCON), Cavtat, Croacia, 2014, pp. 358-365. DOI: https://doi. org/10.1109/ENERGYCON.2014.6850452

[14] G. A. Marrero y F. J. Ramos-Real, "Electricity generation cost in isolated system: The complementarities of natural gas and renewables in the Canary Islands," Renewable and Sustainable Energy Reviews, vol. 14, no. 9, pp. 2808-2818, 2010. DOI: https://doi. org/10.1016/j.rser.2010.06.007

[15] F. J. Santos-Alamillos, D. Pozo-Vázquez, J. A. RuizArias, V. Lara-Fanego y J. Tovar-Pescador, "Analysis of spatiotemporal balancing between wind and solar energy resources in the southern Iberian Peninsula," Journal of Applied Meteorogy and. Climatology, vol. 51, no. 11, pp. 2005-2024, 2012. DOI: https://doi.org/10.1175/ JAMC-D-11-0189.1

[16] E. R. Tello Ortiz y E. Fadigas. Metodología de jerarquización de áreas para proyectos eólicos mediante análisis secuencial de correlación combinatoria hídrica-eólica, Lima, Perú: Luzlop Editorias, 2011.

[17] E. Kahn, "The reliability of distributed wind generators," Electric Power Systems Research, vol. 2, no. 1, pp. 1-14, 1979. DOI: https://doi.org/10.1016/03787796(79)90021-X

[18] L. Landberg, "The vailability and variability of the European wind resource," International Journal of Solar
Energy, vol. 18, no. 4, pp. 313-320, 1997. DOI: https:// doi.org/10.1080/01425919708914326

[19] C. L. Archer y M. Z. Jacobson, "Spatial and temporal distributions of U.S. winds and wind power at $80 \mathrm{~m}$ derived from measurements," Journal of Geophysical Research, vol. 108, no. D9, 2003. DOI: https://doi.org/10.1029/ 2002JD002076

[20] K. Vogstad, "Utilising the complementary characteristics of wind power and hydropower through coordinated hydro production scheduling using the EMPS model," Wind Power 21st century, pp. 1-5, 2000. [En línea]. Disponible en: https://www.researchgate.net/ publication/234165728_Utilising_the_complementary_characteristics_of_wind_power_and_hydropower_throughcoordinated_hydro_production scheduling_using_the_EMPS_model

[21] I. Kougias, S. Szabó, F. Monforti-Ferrario, T. Huld y K. Bódis, "A methodology for optimization of the complementarity between small-hydropower plants and solar PV systems," Renewable Energy, vol. 87, pp. 1023-1030, 2016. DOI: https://doi.org/10.1016/j.renene.2015.09.073

[22] J. Widén, "Correlations between large-scale solar and wind power in a future scenario for Sweden," IEEE Transactions on Sustainable Energy, vol. 2, no. 2, pp. 177-184, 2011.DOI: https://doi.org/10.1109/TSTE.2010.2101620

[23] F. J. Santos-Alamillos, D. Pozo-Vázquez, J. A. RuizArias, L. Von Bremen y J. Tovar-Pescador, "Combining wind farms with concentrating solar plants to provide stable renewable power," Renewable Energy, vol. 76, pp. 539-550, 2015. DOI: https://doi.org/10.1016/j.renene.2014.11.055

[24] D. Rimantas Pranas y S. Aldas, "Modelling and Analysis of Wind and Solar Generation," en 2014 Electric Power Quality and Supply Reliability Conference (PQ). Rakvere, Estonia, 2014, pp. 139-142.

[25] B. François et al., "Complementarity between solar and hydro power: Sensitivity study to climate characteristics in Northern-Italy," Renewable Energy, vol. 86, pp. 543-553, 2016. DOI: https://doi.org/10.1016/j.renene.2015.08.044

[26] M. Huber, D. Dimkova y T. Hamacher, "Integration of wind and solar power in Europe: Assessment of flexibility requirements," Energy, vol. 69, pp. 236-246, 2014. DOI: https://doi.org/10.1016/j.energy.2014.02.109

[27] A. A. Prasad, R. A. Taylor y M. Kay, "Assessment of solar and wind resource synergy in Australia," Applied Energy, vol. 190, pp. 354-367, 2017. DOI: https://doi. org/10.1016/j.apenergy.2016.12.135

[28] S. B. Kim et al., "Correlation Analysis of Renewable Energy Sources for Future Power Preparation in Jeju," 
$A S T L$, vol. 19, pp. 135-138, 2013. [En línea]. Disponible en: http://modul.repo.mercubuana-yogya.ac.id/modul/files/openjournal/JournalOfArt/34_9.pdf

[29] K. Suomalainen, G. Pritchard, B. Sharp, Z. Yuan y G. Zakeri, "Correlation analysis on wind and hydro resources with electricity demand and prices in New Zealand," Applied Energy, vol. 137, pp. 445-462, 2015. DOI: https://doi.org/10.1016/j.apenergy.2014.10.015

[30] S. Sinha y S. S. Chandel, "Prospects of solar photovoltaic-micro-wind based hybrid power systems in western Himalayan state of Himachal Pradesh in India," Energy Conversion and Management, vol. 105, pp. 1340-1351, 2015. DOI: https://doi.org/10.1016/j.enconman.2015.08.078

[31] W. P. Bell, P. Wild, J. Foster y M. Hewson, "Wind speed and electricity demand correlation analysis in the Australian National Electricity Market: Determining wind turbine generators' ability to meet electricity demand without energy storage," Economic Analysis Policy, vol. 48, pp. 182-191, 2015. DOI: https://doi.org/10.1016/j.eap.2015.11.009

[32] W. Li, S. Stadler y R. Ramakumar, "Modeling and Assessment of Wind and Insolation Resources with a Focus on Their Complementary Nature : A Case Study of Oklahoma Modeling and Assessment of Wind and Insolation Resources with a Focus on Their Complementary Nature : A Case Study of Oklahoma," Annals of the Association of American Geographers, vol. 101, no. 4, pp. 37-41, 2010. DOI: https://doi.org/10.1080/00 045608.2011 .567926

[33] C. E. Hoicka y I. H. Rowlands, "Solar and wind resource complementarity: Advancing options for renewable electricity integration in Ontario, Canada," Renewable Energy, vol. 36, no. 1, 2017, pp. 97-107, 2011. DOI: https:// doi.org/10.1016/j.renene.2010.06.004

[34] M. J. Mowers, C. Helm, N. Blair y W. Campbell Short, "Correlations Between Geographically Dispersed Concentrating Solar Power And Demand In The United States," pp. 1-9, 2017. [En línea]. Disponible en: http:// proceedings.asmedigitalcollection.asme.org/proceeding. aspx?articleid $=1607646$

[35] D. B. Richardson y L. D. D. Harvey, "Strategies for correlating solar PV array production with electricity demand," Renewable Energy, vol. 76, pp. 432-440, 2015. DOI: https://doi.org/10.1016/j.renene.2014.11.053

[36] International Renewable Energy Agency (Irena), "Renewable Energy Market Analysis: Latin America," 2016. [En línea]. Disponible en: https://www.irena.org/ DocumentDownloads/Publications/IRENA_Market_ Analysis_Latin_America_2016.pdf
[37] N. José de Castro, G. De, A. D. André, L. Da, S. Leite y J. Goodward, "Perspectivas para a Energia Eólica no Brasil,” 2010. [En línea]. Disponible en: http://www. gesel.ie.ufrj.br/app/webroot/files/publications/01_ tdse18.pdf

[38] G. C. Palfi y R. C. Zambon, "Hydro and Wind Power Complementarity and Scenarization in Brazil," en World Environmental and Water Resources Congress, 2013, pp. 2414-2424. DOI: https://doi.org/10.1061/ 9780784412947.237

[39] V. S. Lopes y C. L. T. Borges, "Impact of the Combined Integration of Wind Generation and Small Hydropower Plants on the System Reliability," IEEE Transactions on Sustainable Energy, vol. 6, no. 3, pp. 1169-1177, 2015. DOI: https://doi.org/10.1109/TSTE.2014.2335895

[40] D. S. Ramos, L. A. S. Camargo, E. Guarnier y L. T. Witzler, "Minimizing market risk by trading hydro-wind portfolio: A complementarity approach," en 2013 10th International Conference on the European Energy Market (EEM), Estocolmo, Siuza, 2013. DOI: https://doi.org/10.1109/EEM.2013.6607300

[41] O. A. Jaramillo, M. A. Borja y J. M. Huacuz, "Using hydropower to complement wind energy: A hybrid system to provide firm power," Renewable Energy, vol. 29, no. 11, pp. 1887-1909, 2004. DOI: https://doi. org/10.1016/j.renene.2004.02.010

[42] A. Beluco, P. Kroeff de Souza y A. Krenzinger, "A method to evaluate the effect of complementarity in time between hydro and solar energy on the performance of hybrid hydro PV generating plants," Renewable Energy, vol. 45, pp. 24-30, 2012. DOI: https://doi. org/10.1016/j.renene.2012.01.096

[43] P. Sales dos Anjos, A. S. Alves da Silva, B. Stošić y T. Stošić, "Long-term correlations and cross-correlations in wind speed and solar radiation temporal series from Fernando de Noronha Island, Brazil," Physica A: Statistical Mechanics and its Applications, vol. 424, pp. 90-96, 2015. DOI: https://doi.org/10.1016/j.physa.2015.01.003

[44] Fundación Julio Ricaldoni Ingeniería en el Uruguay, "Complementariedad de las Energías Renovables en Uruguay y valorización de proyectos para el filtrado de su variabilidad," pp. 1-108, 2014. [En línea]. Disponible en: https://www.miem.gub.uy/sites/default/ files/complementariedad_de_las_energias_renovables_en_uruguay.pdf

[45] Fundación Julio Ricaldoni Ingeniería en el Uruguay et al., "Análisis de complementariedad de los recursos eólico y solar para su utilización en la generación eléctrica en gran escala en Uruguay,” 2016. [En línea]. 
Disponible en: https://iie.fing.edu.uy/publicaciones/2016/ GCDDTAAMALC16/GCDDTAAMALC16.pdf

[46] A. J. Ealo Otero, “Análisis de generación complementaria entre energía hidráulica y eólica caso: generación ISAGEN - proyectos eólicos en la Guajira Colombiana," Tesis de maestría, Universidad nacional de Colombia, Medellín, Colombia, 2011.

[47] C. A. González Manosalva, "Definición de la composición en las fuentes hidráulica y eólica para la generación de energía eléctrica en el contexto colombiano aplicando la teoría de portafolio," Tesis de maestría, Universidad Nacional de Colombia, Medellín, Colombia, 2012.

[48] Banco Interamericano de Desarrollo, Unidad de Planeación Minero Energética (UPME), Ministerio de Minas y Energía y COWI, "Impact analysis for integration of wind power generation in Colombia project report,” 2014. [En línea]. Disponible en: https://www.
neplan.ch/wp-content/uploads/2016/03/38811-Project-Report-Rev-0-29Nov14.pdf

[49] W. Vergara et al. Wind energy in Colombia: a framework for market entry. Washington, D. C., Estados Unidos: World Bank, 2010. DOI: https://doi.org/10.1596/978-08213-8504-3

[50] Unidad de Planeación Minero Energética (UPME), "Formulación de un plan de desarrollo para las fuentes no convencionales de energía en Colombia (PDFNCE)," 2010. [En línea]. Disponible en: http://www. upme.gov.co/sigic/documentosf/vol_3_tecnologia_ costos_fnce.pdf

[51] Unidad de Planeación Minero Energética (UPME), "Plan de Energización Sostenible del Departamento de Cundinamarca - Diagnóstico Energético,” no. 40, 2017. [En línea]. Disponible en: http://egresado.udistrital.edu.co/recursos/Caracterizacion\%20Demanda\%20 PERS\%20Cundinamarca\%20V1.pdf 